\title{
Stabilization of the weakly coupled plate equations with a locally distributed damping
}

Xianzheng Zhu ${ }^{1 *}$ (D)

\section{"Correspondence:}

zhuxzheng@126.com

'School of Mathematics, Sichuan

University, Chengdu, China

\section{Springer}

\begin{abstract}
In this paper, we study the indirect stabilization of a system of plate equations which are weakly coupled and locally damped. By virtue of the general results due to Burq in the study of asymptotic behavior of solutions, we prove that the semigroup associated to the system is logarithmically stable under some assumptions on the damping and the coupling terms. For this purpose, we adopt an approach based on the growth of the resolvent on the imaginary axis, which can be obtained by some Carleman estimates.
\end{abstract}

Keywords: Coupled plate equations; Indirect damping; Logarithmic decay; Carleman estimate

\section{Introduction}

Let $\Omega$ be a bounded domain in $\mathbb{R}^{n}$ with a $C^{4}$ boundary $\Gamma$. Consider the following two weakly coupled plate equations:

$$
\begin{cases}y_{t t}+\Delta^{2} y+c(x) z+d(x) y_{t}=0 & \text { in } \mathbb{R}^{+} \times \Omega, \\ z_{t t}+\Delta^{2} z+c(x) y=0 & \text { in } \mathbb{R}^{+} \times \Omega, \\ y=\Delta y=z=\Delta z=0 & \text { on } \mathbb{R}^{+} \times \Gamma, \\ \left(y(0), y_{t}(0)\right)=\left(y^{0}, y^{1}\right),\left(z(0), z_{t}(0)\right)=\left(z^{0}, z^{1}\right) & \text { in } \Omega,\end{cases}
$$

where $c(\cdot) \in L^{\infty}(\Omega ; \mathbb{R})$ is the coupling function, and $d(\cdot) \in L^{\infty}(\Omega ; \mathbb{R})$ is the damping function. Both $c(\cdot)$ and $d(\cdot)$ are nonnegative.

In system (1.1), the damping which is distributed locally in the domain under consideration acts through one of the equations only, and its effect is transmitted to the other equation through the coupling. Thus, system (1.1) is a special case of the general framework proposed by Russell (see [28]) for the indirect damping problem in elastic systems. Motivated by Russell's work, the indirect stabilization problem for all kinds of coupled systems have been extensively studied (see e.g. $[1-4,10,16,18,23,27,30]$ and the references therein).

It is well known that a single wave equation is exponentially stable if and only if the geometric control condition (GCC for short) is satisfied (see [6]). When the GCC failed,

(c) The Author(s) 2020. This article is licensed under a Creative Commons Attribution 4.0 International License, which permits use sharing, adaptation, distribution and reproduction in any medium or format, as long as you give appropriate credit to the original author(s) and the source, provide a link to the Creative Commons licence, and indicate if changes were made. The images or other third party material in this article are included in the article's Creative Commons licence, unless indicated otherwise in a credit line to the material. If material is not included in the article's Creative Commons licence and your intended use is not permitted by statutory regulation or exceeds the permitted use, you will need to obtain permission directly from the copyright holder. To view a copy of this licence, visit http://creativecommons.org/licenses/by/4.0/. 
Lebeau [22] first derived the logarithmic decay result for wave equations where no geometric restriction was imposed on the damping regions. After that, the logarithmic decay result was extended to many other problems (see $[5,9,19]$ for a single wave or plate) with internal or boundary damping. However, for the indirect stabilization for weakly coupled wave-type equations (wave-wave, wave-plate, etc.), even under some geometric conditions, the exponential stability does not hold (see [3]). There are many results related to the polynomial decay. In this respect, we refer to [2-4] for the indirect stabilization for weakly coupled systems of wave-wave or wave-Petrowsky type, and [17, 24, 25, 29] for the weakly coupled plate-plate equations. Further, we refer to [21] for the uniform decay rates of the coupled wave equation and plate equation with the coupling on the interface, and [15] for the polynomial decay estimate for a single plate equation with local degenerated dissipations.

It should be pointed out that there are few references addressing the logarithmic decay rate for the indirect stabilization for weakly coupled wave-type equations. We refer to [11] for the logarithmic decay rates of the energy of a hyperbolic-parabolic system coupled by an interface, [12] for the logarithmic decay result of the weakly coupled hyperbolic equations, [13] for the logarithmic decay result of the weakly coupled wave-plate equations. As far as we know, there is no reference addressing the asymptotic behavior of the system (1.1).

In this paper, we will show the logarithmic decay property for solutions of the system (1.1). Due to Burq's [8] general results in the study of asymptotic behavior of solutions, it suffices to show some high-frequency estimates with exponential loss on the resolvent. To this aim, we borrow some ideas in [12,13]. In [12], to get the energy decay for a system coupled by two wave equations, one is required to establish an interpolation inequality for a system coupled by two elliptic equations. In [13], to get the energy decay for a system coupled by wave-plate equations, one is required to establish an interpolation inequality for a system coupled by one elliptic and two parabolic equations. In our case, we consider

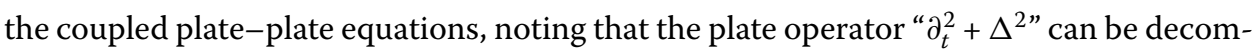
posed as two conjugate Schrödinger ones " $\partial_{t}^{2}+\Delta^{2}=\left(i \partial_{t}+\Delta\right)\left(-i \partial_{t}+\Delta\right)$ ", therefore, we have to get an interpolation inequality for a system coupled by four parabolic-type equations (see (4.5)). Since there is no elliptic-type equation in our situation, the interpolation inequality we obtain here differs from [12, 13]. See Sect. 3 for more details.

The rest of this paper is organized as follows. In Sect. 2, we give the main results in this paper. Section 3 is addressed to proving an interpolation inequality by virtue of Carleman estimates for the parabolic equations. At last, in Sect. 4, we prove our main results.

\section{Statement of the main results}

Let $m^{*}=\inf _{y \in H^{2}(\Omega) \cap H_{0}^{1}(\Omega)}\|\Delta y\|_{L^{2}(\Omega)}^{2} /\|y\|_{L^{2}(\Omega)}^{2}$. Throughout this paper, we assume that $c(\cdot)$ and $d(\cdot)$ satisfy

$$
\left\{\begin{array}{l}
c(x) \geq c_{0}>0 \quad \text { in } \omega_{c} \\
\|c\|_{L^{\infty}(\Omega)}<m^{*}
\end{array}\right.
$$

and

$$
d(x) \geq d_{0}>0 \quad \text { in } \omega_{d},
$$


where $\omega_{c}$ and $\omega_{d}$ are arbitrary non-empty open subsets of $\Omega$. The solutions $y$ and $z$ to system (1.1) are complex-valued functions. In what follows, we shall use $C=$ $C\left(\Omega, \omega_{c}, \omega_{d}, c_{0}, d_{0}\right)$ to denote generic positive constants which may vary from line to line. Now let us introduce the energy space $\mathcal{H}$ over the field $\mathbb{C}$ as follows:

$$
\mathcal{H}=\left(H^{2}(\Omega) \cap H_{0}^{1}(\Omega)\right) \times L^{2}(\Omega) \times\left(H^{2}(\Omega) \cap H_{0}^{1}(\Omega)\right) \times L^{2}(\Omega) .
$$

For any $U=(y, u, z, v), \tilde{U}=(\tilde{y}, \tilde{u}, \tilde{z}, \tilde{v}) \in \mathcal{H}$, their inner product is

$$
(U, \tilde{U}) \triangleq \int_{\Omega}(\Delta y \Delta \overline{\tilde{y}}+\Delta z \Delta \overline{\tilde{z}}+c(x) y \overline{\tilde{z}}+c(x) z \overline{\tilde{y}}) d x+\int_{\Omega}(u \overline{\tilde{u}}+v \overline{\tilde{v}}) d x
$$

Define a linear unbounded operator $\mathcal{A}: D(\mathcal{A}) \subset \mathcal{H} \rightarrow \mathcal{H}$ by

$$
\left\{\begin{array}{l}
D(\mathcal{A})=\left\{U=(y, u, z, v) \in \mathcal{H}: \mathcal{A} U \in \mathcal{H},\left.y\right|_{\Gamma}=\left.\Delta y\right|_{\Gamma}=\left.z\right|_{\Gamma}=\left.\Delta z\right|_{\Gamma}=0\right\} \\
\mathcal{A} U=\left(u,-\Delta^{2} y-c(x) z-d(x) u, v,-\Delta^{2} z-c(x) y\right) .
\end{array}\right.
$$

In fact, if $U=(y, u, z, v) \in D(\mathcal{A})$, we have $y \in H^{4}(\Omega), u \in H^{2}(\Omega) \cap H_{0}^{1}(\Omega), z \in H^{4}(\Omega)$ and $v \in H^{2}(\Omega) \cap H_{0}^{1}(\Omega)$.

Let $U_{0}=\left(y^{0}, y^{1}, z^{0}, z^{1}\right)$. Then system (1.1) can be rewritten as

$$
\frac{d U}{d t}=\mathcal{A} U, \quad U(0)=U_{0}
$$

Proposition 2.1 Assume $\|c\|_{L^{\infty}(\Omega)}<m^{*}$. Then $\mathcal{A}$ is the infinitesimal generator of a $C_{0}$ semigroup of contractions $\left\{e^{t \mathcal{A}}\right\}_{t \geq 0}$ on $\mathcal{H}$. Furthermore, $\mathcal{A}$ has a compact resolvent.

Proof Since $\overline{D(\mathcal{A})}=\mathcal{H}$, by the Lumer-Phillips theorem (see [26]), $\mathcal{A}$ generates a $C_{0}$ semigroup of contractions if $\mathcal{A}$ is dissipative and $0 \in \rho(\mathcal{A})$, where $\rho(\mathcal{A})$ denotes the resolvent set of $\mathcal{A}$. For any $U=(y, u, z, v) \in D(\mathcal{A})$, noting the inner product (2.3) we define on $\mathcal{H}$, it is easy to show that

$$
\operatorname{Re}(\mathcal{A} U, U)=-\int_{\Omega} d(x)|u|^{2} d x \leq 0 .
$$

On the other hand, given $F=\left(f^{0}, f^{1}, g^{0}, g^{1}\right) \in \mathcal{H}$, let us consider the problem $\mathcal{A} U=F$, which is equivalent to

$$
\begin{cases}u=f^{0} & \text { in } \Omega \\ v=g^{0} & \text { in } \Omega\end{cases}
$$

and

$$
\begin{cases}-\Delta^{2} y-c(x) z=d(x) f^{0}+f^{1} & \text { in } \Omega, \\ -\Delta^{2} z-c(x) y=g^{1} & \text { in } \Omega, \\ y=\Delta y=z=\Delta z=0 & \text { on } \Gamma .\end{cases}
$$


Let $\mathcal{G}=\left(H^{2}(\Omega) \cap H_{0}^{1}(\Omega)\right) \times\left(H^{2}(\Omega) \cap H_{0}^{1}(\Omega)\right)$. In the following, we shall prove that the problem (2.6)-(2.7) has a unique solution $U=(y, u, z, v) \in D(\mathcal{A})$. Introduce a sesquiliner form $\Lambda$ over the product space $\mathcal{G} \times \mathcal{G}$ as follows:

$$
\Lambda(V, \tilde{V})=\int_{\Omega}(\Delta y \Delta \overline{\tilde{y}}+\Delta z \Delta \overline{\tilde{z}}+c(x) y \overline{\tilde{z}}+c(x) z \overline{\tilde{y}}) d x
$$

where $V=(y, z), \tilde{V}=(\tilde{y}, \tilde{z}) \in \mathcal{G}$. It is easy to see that $\Lambda$ is continuous and coercive. Thus, by the Lax-Milgram theorem, for $\left(-d(x) f^{0}-f^{1},-g^{1}\right) \in \mathcal{G}^{\prime}$, the system (2.7) has a unique weak solution $(y, z) \in \mathcal{G}$ such that, for any $(\tilde{y}, \tilde{z}) \in \mathcal{G}$,

$$
\int_{\Omega}(\Delta y \Delta \overline{\tilde{y}}+\Delta z \Delta \overline{\tilde{z}}+c(x) y \overline{\tilde{z}}+c(x) z \overline{\tilde{y}}) d x=-\int_{\Omega}\left(d(x) f^{0} \overline{\tilde{y}}+f^{1} \overline{\tilde{y}}+g^{1} \overline{\tilde{z}}\right) d x .
$$

Denote by $\mathcal{D}^{\prime}(\Omega)$ the space of all distributions on $\Omega$. By the equality above, one has

$$
\Delta^{2} y+c(x) z=-d(x) f^{0}-f^{1} \quad \text { in } \mathcal{D}^{\prime}(\Omega)
$$

which implies $\Delta^{2} y=-c(x) z-d(x) f^{0}-f^{1} \in L^{2}(\Omega)$. Let $\tilde{f}=c(x) z+d(x) f^{0}+f^{1}$. Then there exists a unique solution $\vartheta \in H^{2}(\Omega) \cap H_{0}^{1}(\Omega)$ such that $\Delta \vartheta=\tilde{f}$ in $\Omega$ and $\vartheta=0$ on $\Gamma$. Now, noting (2.9), for any $\tilde{y} \in H^{2}(\Omega) \cap H_{0}^{1}(\Omega)$ we have $\int_{\Omega} \vartheta \Delta \tilde{y} d x=\int_{\Omega} \tilde{f} \tilde{y} d x=-\int_{\Omega} \Delta y \Delta \tilde{y} d x$. Since the mapping $-\Delta: H^{2}(\Omega) \cap H_{0}^{1}(\Omega) \rightarrow L^{2}(\Omega)$ is surjective, it follows that $\Delta y=-\vartheta \in$ $H^{2}(\Omega) \cap H_{0}^{1}(\Omega)$. Then, by the elliptic regularity theory, we have $y \in H^{4}(\Omega)$ and the following estimate holds:

$$
\|y\|_{H^{4}(\Omega)} \leq C\left(\|\Delta y\|_{H^{2}(\Omega)}+\|y\|_{L^{2}(\Omega)}\right) .
$$

Thus, combining $\Delta(\Delta y) \in L^{2}(\Omega)$ with $\left.\Delta y\right|_{\Gamma}=0$, by the elliptic regularity theory, once again we have

$$
\|\Delta y\|_{H^{2}(\Omega)} \leq C\left(\|\Delta y\|_{L^{2}(\Omega)}+\left\|c(x) z+d(x) u+f^{1}\right\|_{L^{2}(\Omega)}\right) .
$$

Similarly, we also have $z \in H^{4}(\Omega), \Delta z \in H_{0}^{1}(\Omega)$, and the following estimates hold:

$$
\|z\|_{H^{4}(\Omega)} \leq C\left(\|\Delta z\|_{H^{2}(\Omega)}+\|z\|_{L^{2}(\Omega)}\right)
$$

and

$$
\|\Delta z\|_{H^{2}(\Omega)} \leq C\left(\|\Delta z\|_{L^{2}(\Omega)}+\left\|c(x) y+g^{1}\right\|_{L^{2}(\Omega)}\right) .
$$

On the other hand, taking $(\tilde{y}, \tilde{z})=(y, z)$ in $(2.9)$, it is easy to find that

$$
\|\Delta y\|_{L^{2}(\Omega)}+\|\Delta z\|_{L^{2}(\Omega)} \leq C\|F\|_{\mathcal{H}} .
$$

Finally, combining (2.6) with (2.11)-(2.15) we conclude that $0 \in \rho(\mathcal{A})$ and

$$
\|y\|_{H^{4}(\Omega)}+\|u\|_{H^{2}(\Omega)}+\|z\|_{H^{4}(\Omega)}+\|v\|_{H^{2}(\Omega)} \leq C\|F\|_{\mathcal{H}},
$$

which implies that $\mathcal{A}^{-1}$ is compact. 
The energy of system (1.1) at time $t$ is given by

$$
E(t)=\frac{1}{2} \int_{\Omega}\left(|\Delta y(t)|^{2}+\left|y_{t}(t)\right|^{2}+|\Delta z(t)|^{2}+\left|z_{t}(t)\right|^{2}\right) d x+\int_{\Omega} c(x) \operatorname{Re}(y \bar{z}) d x
$$

When $d \equiv 0, E(\cdot)$ is obviously conservative. Otherwise, we have

$$
E\left(t_{2}\right)-E\left(t_{1}\right)=-\int_{t_{1}}^{t_{2}} \int_{\Omega} d(x)\left|y_{t}\right|^{2} d x d t, \quad \forall t_{2} \geq t_{1} \geq 0
$$

which implies the energy is nonincreasing and system (1.1) is dissipative.

Our main result is stated as follows.

Theorem 2.1 Let $c(\cdot)$ and $d(\cdot)$ satisfy (2.1) and (2.2), respectively. Suppose that $\omega_{c} \cap \omega_{d} \neq$ $\emptyset$. Then there exists a constant $C>0$ such that for all $\left(y^{0}, y^{1}, z^{0}, z^{1}\right) \in D(\mathcal{A})$ the solution $e^{t \mathcal{A}}\left(y^{0}, y^{1}, z^{0}, z^{1}\right) \in C\left(\mathbb{R}^{+} ; D(\mathcal{A})\right) \cap C^{1}\left(\mathbb{R}^{+} ; \mathcal{H}\right)$ to system $(1.1)$ satisfies

$$
\left\|e^{t \mathcal{A}}\left(y^{0}, y^{1}, z^{0}, z^{1}\right)\right\|_{\mathcal{H}} \leq \frac{C}{\ln (2+t)}\left\|\left(y^{0}, y^{1}, z^{0}, z^{1}\right)\right\|_{D(\mathcal{A})}, \quad \forall t \geq 0 .
$$

In order to prove Theorem 2.1, let us recall the following result:

Lemma 2.1 ([7, Theorem A]) Assume that $\mathcal{B}$ is the infinitesimal generator of a bounded $C_{0}$ semigroup $\left\{e^{t \mathcal{B}}\right\}_{t \in \mathbb{R}^{+}}$on Hilbert space $\tilde{\mathcal{H}}$. Let $\rho(\mathcal{B})$ denote the resolvent set of $\mathcal{B}$. If $i \mathbb{R} \subset \rho(\mathcal{B})$ and there exists a positive constant $C$ such that

$$
\sup _{|\tau| \leq \xi}\left\|(i \tau-\mathcal{B})^{-1}\right\|_{\mathcal{L}(\tilde{\mathcal{H}})} \leq C e^{C \xi}, \quad \forall \xi \geq 0
$$

then, for any $k \in \mathbb{N}^{*}$, there exists a positive constant $C_{k}$ such that

$$
\sup _{s \geq t}\left\|e^{s \mathcal{B}}(1-\mathcal{B})^{-k}\right\|_{\mathcal{L}(\tilde{\mathcal{H}})} \leq \frac{C_{k}}{\ln ^{k}(2+t)}, \quad \forall t \geq 0
$$

where $\mathbb{N}^{*}=\mathbb{N} \backslash\{0\}$.

According to Lemma 2.1, Theorem 2.1 is reduced to the following resolvent estimate of the operator $\mathcal{A}$.

Theorem 2.2 Under the assumptions of Theorem 2.1, we have $i \mathbb{R} \subset \rho(\mathcal{A})$ and there exists a constant $C>0$ such that, for every $\beta \in \mathbb{R}$,

$$
\left\|(i \beta-\mathcal{A})^{-1}\right\|_{\mathcal{L}(\mathcal{H})} \leq C e^{C|\beta|}
$$

Remark 2.1 In this paper, we assume that $\omega_{c} \cap \omega_{d} \neq \emptyset$. It would be quite interesting to consider the case that $\omega_{c} \cap \omega_{d}=\emptyset$. Some results are obtained in [4] for one dimensional coupled wave equations. For the multi-dimensional case, as far as we know, it is an unsolved problem. 


\section{An interpolation inequality for the coupled parabolic system}

In this section, we shall prove an interpolation inequality for the following coupled parabolic system:

$$
\begin{cases}p_{s}+\Delta p=w & \text { in } Q, \\ w_{s}-\Delta w-i d(x) p_{s}-c(x) q=w^{0} & \text { in } Q, \\ q_{s}+\Delta q=z & \text { in } Q, \\ z_{s}-\Delta z-c(x) p=z^{0} & \text { in } Q, \\ p=\Delta p=q=\Delta q=0 & \text { on } \Sigma .\end{cases}
$$

Here $Q=(-2,2) \times \Omega, \Sigma=(-2,2) \times \Gamma$ and $w^{0}, z^{0} \in L^{2}(Q)$.

Set

$$
X \triangleq(-1,1) \times \Omega, \quad \tilde{X} \triangleq(-2,2) \times \omega, \quad \omega=\omega_{c} \cap \omega_{d} .
$$

We have the following interpolation inequality for the system (3.1).

Theorem 3.1 Under the assumptions in Theorem 2.1, there exists a constant $C>0$ such that, for any $\varepsilon>0$, any solution $(p, w, q, z)$ of the system (3.1) satisfies

$$
\begin{aligned}
& \|p\|_{L^{2}(X)}+\|w\|_{L^{2}(X)}+\|q\|_{L^{2}(X)}+\|z\|_{L^{2}(X)} \\
& \leq C e^{C / \varepsilon}\left(\left\|w^{0}\right\|_{L^{2}(Q)}+\left\|z^{0}\right\|_{L^{2}(Q)}+\|p\|_{L^{2}(\widetilde{X})}+\left\|d p_{s}\right\|_{L^{2}(Q)}\right) \\
& +C e^{-1 / \varepsilon}\left(\|p\|_{L^{2}(Q)}+\|w\|_{L^{2}(Q)}+\|q\|_{L^{2}(Q)}+\|z\|_{L^{2}(Q)}\right) .
\end{aligned}
$$

\subsection{Some preliminaries}

The proof of Theorem 3.1 is based on the Carleman estimates for the parabolic operators $\pm \partial_{s}+\Delta$. In this subsection, we collect some known results we need.

As we know, Carleman estimate can be regarded as a weighted energy estimate. To begin with, we first give the choice of the weight functions. Let $\omega_{0}$ be a non-empty subdomain of $\Omega$ such that $\omega_{0} \subset \bar{\omega}_{0} \subset \omega$. By [14, Lemma 1.1] we know that there exists a function $\hat{\psi} \in C^{2}(\bar{\Omega} ; \mathbb{R})$ such that

$$
\hat{\psi}>0 \quad \text { in } \Omega, \quad \hat{\psi}=0 \quad \text { on } \partial \Omega, \quad|\nabla \hat{\psi}|>0 \quad \text { in } \overline{\Omega \backslash \omega_{0}} .
$$

With such choice of $\hat{\psi}$ and $\lambda, \mu>1$, we define

$$
\left\{\begin{array}{l}
\psi=\psi(s, x) \triangleq \frac{\hat{\psi}(x)}{\|\hat{\psi}\|_{L^{\infty}(\Omega)}}+b^{2}-s^{2}, \quad x \in \bar{\Omega}, s \in \mathbb{R}, \\
\theta=e^{\ell}, \quad \ell=\lambda \phi, \quad \phi=e^{\mu \psi},
\end{array}\right.
$$

where $b \in(1,2)$ will be given later.

Choose a cut-off function $\eta \in C_{0}^{\infty}(\omega)$ such that

$$
\begin{cases}0 \leq \eta(x) \leq 1, & x \in \omega \\ \eta(x)=1, & x \in \omega_{0} .\end{cases}
$$


For fixed $\mu$, proceeding with exactly the same analysis as [13], we have the following result.

Lemma 3.1 ([13, Lemma 3.2]) Let $\gamma \in \mathbb{R}$ and $\ell$ be given by (3.4). Let $k$ be a positive integer and $k \geq 2$. Then, for a fixed $\mu$, there is a constant $\lambda_{0}>0$ such that, for all $\lambda \geq \lambda_{0}$, one can find a constant $C>0$ such that, for all $z \in H_{0}^{2}\left(-b, b ; H^{2}(\Omega) \cap H_{0}^{1}(\Omega)\right)$ and for any $\beta \geq 2$,

$$
\begin{aligned}
& \int_{-b}^{b} \int_{\Omega} \theta^{2} \eta^{k}|\nabla z|^{2} d x d s \\
& \quad \leq \frac{1}{\lambda^{\beta}} \int_{-b}^{b} \int_{\Omega} \theta^{2} \eta^{k}\left|\gamma z_{s}+\Delta z\right|^{2} d x d s+C \lambda^{\beta} \int_{-b}^{b} \int_{\Omega} \theta^{2} \eta^{k-2}|z|^{2} d x d s .
\end{aligned}
$$

Further, we recall the following well-known Carleman estimate for the parabolic operator $\gamma z_{s}+\Delta z$.

Lemma 3.2 ([13, Lemma 3.4]) Let $\gamma \in \mathbb{R}$ and $\ell$ be given by (3.4). Then there is a constant $\mu_{1}>0$ such that, for all $\mu \geq \mu_{1}$, one can find two constants $C>0$ and $\lambda_{1}=\lambda_{1}(\mu)>0$ so that, for any $\lambda \geq \lambda_{1}$, for all $z \in H_{0}^{2}\left(-b, b ; H^{2}(\Omega) \cap H_{0}^{1}(\Omega)\right)$,

$$
\begin{aligned}
& \lambda \mu^{2} \int_{-b}^{b} \int_{\Omega} \theta^{2} \phi\left(|\nabla z|^{2}+\lambda^{2} \mu^{2} \phi^{2}|z|^{2}\right) d x d s \\
& \quad \leq C\left(\int_{-b}^{b} \int_{\Omega} \theta^{2}\left|\gamma z_{s}+\Delta z\right|^{2} d x d s+\lambda^{3} \mu^{4} \int_{-b}^{b} \int_{\omega_{0}} \theta^{2} \phi^{3}|z|^{2} d x d s\right) .
\end{aligned}
$$

\subsection{Proof of Theorem 3.1}

To prove the interpolation inequality (3.2), first we apply the Carleman estimates in Lemma 3.2 to $p, w, q$ and $z$, respectively, to get (3.13). Then the main difficulty is to estimate the energy of $(\hat{p}, \hat{q}, \hat{w}, \hat{z})$ localized on $\omega_{0}$ by only the energy of $p$ localized on $\omega$, which can be solved by using their coupling relations and the multiplier technique. Since the proof is long, we divide it into several steps.

Step 1. There is no boundary conditions for $p, w, q$ and $z$ at $s= \pm 2$ in the system (3.1). Thus, we need to introduce a cut-off function $\varphi=\varphi(s) \in C_{0}^{\infty}(-b, b)$ such that

$$
\begin{cases}0 \leq \varphi(s) \leq 1, & |s|<b \\ \varphi(s)=1, & |s| \leq b_{0}\end{cases}
$$

Here $1<b_{0}<b \leq 2$ are given as follows:

$$
b \triangleq \sqrt{1+\frac{1}{\mu} \ln \left(2+e^{\mu}\right)}, \quad b_{0} \triangleq \sqrt{b^{2}-\frac{1}{\mu} \ln \left(\frac{1+e^{\mu}}{e^{\mu}}\right)},
$$

where $\mu$ is the parameter appeared in Lemma 3.2 and is large enough. Put

$$
\hat{p}=\varphi p, \quad \hat{w}=\varphi w, \quad \hat{q}=\varphi q, \quad \hat{z}=\varphi z .
$$


Noting that $\varphi$ does not depend on $x$, it follows from (3.1) that

$$
\begin{cases}\hat{p}_{s}+\Delta \hat{p}=F_{1} & \text { in } Q, \\ \hat{w}_{s}-\Delta \hat{w}=F_{2} & \text { in } Q, \\ \hat{q}_{s}+\Delta \hat{q}=F_{3} & \text { in } Q, \\ \hat{z}_{s}-\Delta \hat{z}=F_{4} & \text { in } Q, \\ \hat{p}=\Delta \hat{p}=\hat{q}=\Delta \hat{q}=0 & \text { on } \Sigma,\end{cases}
$$

where

$$
\left\{\begin{array}{l}
F_{1} \triangleq \hat{w}+\varphi_{s} p \\
F_{2} \triangleq i d \hat{p}_{s}+c(x) \hat{q}+\varphi_{s} w-i d \varphi_{s} p+\varphi w^{0}, \\
F_{3} \triangleq \hat{z}+\varphi_{s} q \\
F_{4}=c(x) \hat{p}+\varphi_{s} z+\varphi z^{0} .
\end{array}\right.
$$

By using Lemma 3.2 for $\gamma= \pm 1$, we conclude that there is a $\mu_{1}>0$ such that, for all $\mu \geq \mu_{1}$, one can find two constants $C=C(\mu)>0$ and $\lambda_{1}=\lambda_{1}(\mu)$ so that, for all $\lambda \geq \lambda_{1}$,

$$
\begin{aligned}
\lambda \mu^{2} \int_{-b}^{b} \int_{\Omega} \theta^{2} \phi\left(|\nabla \hat{p}|^{2}+|\nabla \hat{w}|^{2}+|\nabla \hat{q}|^{2}+|\nabla \hat{z}|^{2}\right. \\
\left.\quad+\lambda^{2} \mu^{2} \phi^{2}\left(|\hat{p}|^{2}+|\hat{w}|^{2}+|\hat{q}|^{2}+|\hat{z}|^{2}\right)\right) d x d s \\
\leq C\left[\int_{-b}^{b} \int_{\Omega} \theta^{2}\left(\left|F_{1}\right|^{2}+\left|F_{2}\right|^{2}+\left|F_{3}\right|^{2}+\left|F_{4}\right|^{2}\right) d x d s\right. \\
\left.\quad+\lambda^{3} \mu^{4} \int_{-b}^{b} \int_{\omega_{0}} \theta^{2} \phi^{3}\left(|\hat{p}|^{2}+|\hat{w}|^{2}+|\hat{q}|^{2}+|\hat{z}|^{2}\right) d x d s\right] .
\end{aligned}
$$

Step 2. Let us estimate $\int_{-b}^{b} \int_{\omega_{0}} \theta^{2}|\hat{z}|^{2} d x d s$.

Recall that $\eta \in C_{0}^{\infty}(\omega)$ satisfying $\eta=1$ in $\omega_{0}$. By (3.11) and (3.12), we have

$$
\begin{aligned}
\theta^{2} \eta^{12}|\hat{z}|^{2} \\
=\theta^{2} \eta^{12} \overline{\hat{z}}\left(\hat{q}_{s}+\Delta \hat{q}\right)-\theta^{2} \eta^{12} \overline{\hat{z}} \varphi_{s} q \\
=-\theta^{2} \eta^{12} \hat{q}\left(\overline{\hat{z}}_{s}-\Delta \overline{\hat{z}}\right)+\left(\theta^{2} \eta^{12} \overline{\hat{z}} \hat{q}\right)_{s}-\left(\theta^{2} \eta^{12}\right)_{s} \overline{\hat{z}} \hat{q}-\theta^{2} \eta^{12} \overline{\hat{z}} \varphi_{s} q \\
\quad+\sum_{j=1}^{n}\left[\theta^{2} \eta^{12}\left(\overline{\hat{z}} \hat{q}_{x_{j}}-\overline{\hat{z}}_{x_{j}} \hat{q}\right)\right]_{x_{j}}+\sum_{j=1}^{n}\left(\theta^{2} \eta^{12}\right)_{x_{j}}\left(\overline{\hat{z}}_{x_{j}} \hat{q}-\overline{\hat{z}} \hat{q}_{x_{j}}\right) .
\end{aligned}
$$

Integrating (3.14) on $(-b, b) \times \Omega$, noting that $\hat{z}(-b)=\hat{z}(b)=0$ in $\Omega, \hat{q}=\hat{z}=0$ on the boundary, by (3.11)-(3.12), we find that

$$
\begin{aligned}
& \int_{-b}^{b} \int_{\Omega} \theta^{2} \eta^{12}|\hat{z}|^{2} d x d s \\
& \quad \leq C\left[\int_{-b}^{b} \int_{\Omega} \theta^{2}\left|z^{0}\right|^{2} d x d s+\int_{-b}^{b} \int_{\omega} \theta^{2}|\hat{p}|^{2} d x d s\right.
\end{aligned}
$$


Thu Advances in Difference Equations

(2020) 2020:230

Page 9 of 15

$$
\begin{aligned}
& +\int_{\left(-b,-b_{0}\right) \cup\left(b_{0}, b\right)} \int_{\Omega} \theta^{2}\left(|q|^{2}+|z|^{2}\right) d x d s+\lambda^{5} \int_{-b}^{b} \int_{\Omega} \theta^{2} \eta^{11}|\hat{q}|^{2} d x d s \\
& \left.+\lambda^{2} \int_{-b}^{b} \int_{\Omega} \theta^{2} \eta^{10}|\nabla \hat{q}|^{2} d x d s+\frac{1}{\lambda^{3}} \int_{-b}^{b} \int_{\Omega} \theta^{2}|\nabla \hat{z}|^{2}\right] .
\end{aligned}
$$

Next, taking $\beta=3$ in Lemma 3.1, we have

$$
\begin{aligned}
& \int_{-b}^{b} \int_{\Omega} \theta^{2} \eta^{10}|\nabla \hat{q}|^{2} d x d s \\
& \quad \leq \frac{1}{\lambda^{3}} \int_{-b}^{b} \int_{\Omega} \theta^{2} \eta^{10}\left|F_{3}\right|^{2} d x d s+C \lambda^{3} \int_{-b}^{b} \int_{\Omega} \theta^{2} \eta^{8}|\hat{q}|^{2} d x d s .
\end{aligned}
$$

Combining (3.15) and (3.16) we obtain

$$
\begin{aligned}
\int_{-b}^{b} \int_{\Omega} & \theta^{2} \eta^{12}|\hat{z}|^{2} d x d s \\
\leq & C\left[\int_{-b}^{b} \int_{\Omega} \theta^{2}\left|z^{0}\right|^{2} d x d s+\int_{-b}^{b} \int_{\omega} \theta^{2}|\hat{p}|^{2} d x d s\right. \\
& +\int_{\left(-b,-b_{0}\right) \cup\left(b_{0}, b\right)} \int_{\Omega} \theta^{2}\left(|q|^{2}+|z|^{2}\right) d x d s \\
& \left.+\lambda^{5} \int_{-b}^{b} \int_{\Omega} \theta^{2} \eta^{8}|\hat{q}|^{2} d x d s+\frac{1}{\lambda^{3}} \int_{-b}^{b} \int_{\Omega} \theta^{2}\left(|\nabla \hat{z}|^{2}+\lambda^{2}|\hat{z}|^{2}\right) d x d s\right] .
\end{aligned}
$$

Step 3. Let us estimate $\int_{-b}^{b} \int_{\Omega} \theta^{2} \eta^{8}|\hat{q}|^{2} d x d s$.

Multiplying the second equation of (3.11) by $\theta^{2} \eta^{8} \overline{\hat{q}}$, we get

$$
\begin{aligned}
c(x) & \theta^{2} \eta^{8}|\hat{q}|^{2} \\
= & \theta^{2} \eta^{8} \overline{\hat{q}}\left(\hat{w}_{s}-\Delta \hat{w}\right)-\theta^{2} \eta^{8} \overline{\hat{q}}\left(i d \hat{p}_{s}+\varphi_{s} w-i d \varphi_{s} p+\varphi w^{0}\right) \\
= & -\theta^{2} \eta^{8} \hat{w}\left(\overline{\hat{q}}_{s}+\Delta \overline{\hat{q}}\right)+\left(\theta^{2} \eta^{8} \overline{\hat{q}} \hat{w}\right)_{s}-\left(\theta^{2} \eta^{8}\right)_{s} \overline{\hat{q}} \hat{w}-\theta^{2} \eta^{8} \overline{\hat{q}}\left(i d \varphi p_{s}+\varphi_{s} w+\varphi w^{0}\right) \\
& -\sum_{j=1}^{n}\left[\theta^{2} \eta^{8}\left(\overline{\hat{q}} \hat{w}_{x_{j}}-\overline{\hat{q}}_{x_{j}} \hat{w}\right)\right]_{x_{j}}-\sum_{j=1}^{n}\left(\theta^{2} \eta^{8}\right)_{x_{j}}\left(\overline{\hat{q}}_{x_{j}} \hat{w}-\overline{\hat{q}} \hat{w}_{x_{j}}\right) .
\end{aligned}
$$

Now, integrating (3.18) on $(-b, b) \times \Omega$, recalling (2.1) for $c_{0}$, we find that

$$
\begin{aligned}
& c_{0} \int_{-b}^{b} \int_{\Omega} \theta^{2} \eta^{8}|\hat{q}|^{2} d x d s \\
& \leq C {\left[\int_{-b}^{b} \int_{\Omega} \theta^{2}\left|w^{0}\right|^{2} d x d s+\int_{-b}^{b} \int_{\omega} \theta^{2}\left|p_{s}\right|^{2} d x d s\right.} \\
&+\int_{\left(-b,-b_{0}\right) \cup\left(b_{0}, b\right)} \int_{\Omega} \theta^{2}\left(|w|^{2}+|q|^{2}\right) d x d s \\
&+\lambda^{10} \int_{-b}^{b} \int_{\Omega} \theta^{2} \eta^{7}|\hat{w}|^{2} d x d s+\lambda^{2} \int_{-b}^{b} \int_{\Omega} \theta^{2} \eta^{6}|\nabla \hat{w}|^{2} d x d s \\
&\left.+\frac{1}{\lambda^{8}} \int_{-b}^{b} \int_{\Omega} \theta^{2}\left(|\nabla \hat{q}|^{2}+\lambda^{2}|\hat{z}|^{2}\right)\right] .
\end{aligned}
$$


By Lemma 3.1 and taking $\beta=8$, we have

$$
\begin{aligned}
& \int_{-b}^{b} \int_{\Omega} \theta^{2} \eta^{6}|\nabla \hat{w}|^{2} d x d s \\
& \quad \leq \frac{1}{\lambda^{8}} \int_{-b}^{b} \int_{\Omega} \theta^{2} \eta^{6}\left|F_{2}\right|^{2} d x d s+C \lambda^{8} \int_{-b}^{b} \int_{\Omega} \theta^{2} \eta^{4}|\hat{w}|^{2} d x d s
\end{aligned}
$$

Substituting (3.20) into (3.19), it is easy to show

$$
\begin{aligned}
& \int_{-b}^{b} \int_{\Omega} \theta^{2} \eta^{8}|\hat{q}|^{2} d x d s \\
& \leq C\left[\int_{-b}^{b} \int_{\Omega} \theta^{2}\left|w^{0}\right|^{2} d x d s+\int_{-b}^{b} \int_{\omega} \theta^{2}\left|p_{s}\right|^{2} d x d s\right. \\
&+\int_{\left(-b,-b_{0}\right) \cup\left(b_{0}, b\right)} \int_{\Omega} \theta^{2}\left(|w|^{2}+|q|^{2}\right) d x d s \\
&\left.+\lambda^{10} \int_{-b}^{b} \int_{\Omega} \theta^{2} \eta^{4}|\hat{w}|^{2} d x d s+\frac{1}{\lambda^{8}} \int_{-b}^{b} \int_{\Omega} \theta^{2}\left(|\nabla \hat{q}|^{2}+\lambda^{2}|\hat{z}|^{2}+\lambda^{2}|\hat{q}|^{2}\right)\right]
\end{aligned}
$$

Step 4. Let us estimate $\int_{-b}^{b} \int_{\Omega} \theta^{2} \eta^{4}|\hat{w}|^{2} d x d s$.

Similar to (3.14), we have

$$
\begin{aligned}
\theta^{2} \eta^{4}|\hat{w}|^{2} \\
=\theta^{2} \eta^{4} \overline{\hat{w}}\left(\hat{p}_{s}+\Delta \hat{p}\right)-\theta^{2} \eta^{4} \overline{\hat{w}} \varphi_{s} p \\
=-\theta^{2} \eta^{4} \hat{p}\left(\overline{\hat{w}}_{s}-\Delta \overline{\hat{w}}\right)+\left(\theta^{2} \eta^{4} \overline{\hat{w}} \hat{p}\right)_{s}-\left(\theta^{2} \eta^{4}\right)_{s} \overline{\hat{w}} \hat{p}-\theta^{2} \eta^{4} \overline{\hat{w}} \varphi_{s} p \\
\quad+\sum_{j=1}^{n}\left[\theta^{2} \eta^{4}\left(\overline{\hat{w}} \hat{p}_{x_{j}}-\overline{\hat{w}}_{x_{j}} \hat{p}\right)\right]_{x_{j}}+\sum_{j=1}^{n}\left(\theta^{2} \eta^{4}\right)_{x_{j}}\left(\overline{\hat{w}}_{x_{j}} \hat{p}-\overline{\hat{w}} \hat{p}_{x_{j}}\right) .
\end{aligned}
$$

Integrating (3.22) on $(-b, b) \times \Omega$, we find that

$$
\begin{aligned}
\int_{-b}^{b} \int_{\Omega} & \theta^{2} \eta^{4}|\hat{w}|^{2} d x d s \\
\leq & C\left[\lambda^{20} \int_{-b}^{b} \int_{\omega} \theta^{2}|p|^{2} d x d s+\int_{-b}^{b} \int_{\omega} \theta^{2}\left|p_{s}\right|^{2} d x d s\right. \\
& +\int_{\left(-b,-b_{0}\right) \cup\left(b_{0}, b\right)} \int_{\Omega} \theta^{2}|w|^{2} d x d s \\
& +\int_{-b}^{b} \int_{\Omega} \theta^{2}\left|w^{0}\right|^{2} d x d s+\lambda^{2} \int_{-b}^{b} \int_{\Omega} \theta^{2} \eta^{2}|\nabla \hat{p}|^{2} d x d s \\
& \left.+\frac{1}{\lambda^{18}} \int_{-b}^{b} \int_{\Omega} \theta^{2}\left(|\nabla \hat{w}|^{2}+\lambda^{2}|\hat{q}|^{2}\right)\right]
\end{aligned}
$$

Applying Lemma 3.1 again, we deduce

$$
\int_{-b}^{b} \int_{\Omega} \theta^{2} \eta^{2}|\nabla \hat{p}|^{2} d x d s \leq \frac{1}{\lambda^{18}} \int_{-b}^{b} \int_{\Omega} \theta^{2}|\hat{w}|^{2} d x d s+C \lambda^{18} \int_{-b}^{b} \int_{\omega} \theta^{2}|p|^{2} d x d s
$$


By (3.23)-(3.24) we get

$$
\begin{aligned}
\int_{-b}^{b} \int_{\Omega} \theta^{2} \eta^{4}|\hat{w}|^{2} d x d s \\
\leq C\left[\lambda^{20} \int_{-b}^{b} \int_{\omega} \theta^{2}|p|^{2} d x d s+\int_{-b}^{b} \int_{\omega} \theta^{2}\left|p_{s}\right|^{2} d x d s\right. \\
+\int_{\left(-b,-b_{0}\right) \cup\left(b_{0}, b\right)} \int_{\Omega} \theta^{2}|w|^{2} d x d s+\int_{-b}^{b} \int_{\Omega} \theta^{2}\left|w^{0}\right|^{2} d x d s \\
\left.+\frac{1}{\lambda^{18}} \int_{-b}^{b} \int_{\Omega} \theta^{2}\left(|\nabla \hat{w}|^{2}+\lambda^{2}|\hat{q}|^{2}+\lambda^{2}|\hat{w}|^{2}\right)\right] .
\end{aligned}
$$

Step 5. Combining (3.13), (3.17), (3.21) and (3.25), by (3.10), we have

$$
\begin{aligned}
\lambda \mu^{2} & \int_{-1}^{1} \int_{\Omega} \theta^{2} \phi\left(|\nabla p|^{2}+|\nabla w|^{2}+|\nabla q|^{2}+|\nabla z|^{2}\right. \\
& \left.+\lambda^{2} \mu^{2} \phi^{2}\left(|p|^{2}+|w|^{2}+|q|^{2}+|z|^{2}\right)\right) d x d s \\
\leq & C e^{C \lambda}\left[\int_{-b}^{b} \int_{\Omega}\left(\left|z^{0}\right|^{2}+\left|w^{0}\right|^{2}+\left|d p_{s}\right|^{2}\right) d x d s+\int_{-b}^{b} \int_{\omega}|p|^{2} d x d s\right] \\
& +C \lambda^{18} \int_{\left(-b,-b_{0}\right) \cup\left(b_{0}, b\right)} \int_{\Omega} \theta^{2}\left(|p|^{2}+|w|^{2}+|q|^{2}+|z|^{2}\right) d x d s .
\end{aligned}
$$

Recalling (3.4) and (3.9) for the definitions of $\phi, b$ and $b_{0}$, it is easy to see that

$$
\begin{cases}\phi(s, \cdot) \geq 2+e^{\mu}, & \text { for }|s| \leq 1 \\ \phi(s, \cdot) \leq 1+e^{\mu}, & \text { for } b_{0} \leq|s| \leq b\end{cases}
$$

Fixing the parameter $\mu$ in (3.9), and using (3.27), one finds that

$$
\begin{aligned}
\lambda e^{2 \lambda\left(2+e^{\mu}\right)} \int_{-1}^{1} \int_{\Omega}\left(|\nabla p|^{2}+|\nabla w|^{2}+|\nabla q|^{2}+|\nabla z|^{2}+|p|^{2}+|w|^{2}+|q|^{2}+|z|^{2}\right) d x d s \\
\leq C e^{C \lambda}\left[\int_{-2}^{2} \int_{\Omega}\left(\left|z^{0}\right|^{2}+\left|w^{0}\right|^{2}+\left|d p_{s}\right|^{2}\right) d x d s+\int_{-2}^{2} \int_{\omega}|p|^{2} d x d s\right] \\
+C \lambda^{18} e^{2 \lambda\left(1+e^{\mu}\right)} \int_{-2}^{2} \int_{\Omega}\left(|p|^{2}+|w|^{2}+|q|^{2}+|z|^{2}\right) d x d s
\end{aligned}
$$

which implies

$$
\begin{aligned}
& \int_{-1}^{1} \int_{\Omega}\left(|p|^{2}+|w|^{2}+|q|+|z|^{2}\right) d x d s \\
& \leq C e^{C \lambda}\left[\int_{-2}^{2} \int_{\Omega}\left(\left|z^{0}\right|^{2}+\left|w^{0}\right|^{2}+\left|d p_{s}\right|^{2}\right) d x d s+\int_{-2}^{2} \int_{\omega}|p|^{2} d x d s\right] \\
& \quad+C e^{-\lambda} \int_{-2}^{2} \int_{\Omega}\left(|p|^{2}+|w|^{2}+|q|^{2}+|z|^{2}\right) d x d s .
\end{aligned}
$$

For every $\varepsilon>0$, by taking $\lambda$ in (3.29) large enough, it follows that (3.2) holds. Thus we complete the proof of Theorem 3.1. 


\section{Proof of the main result}

In this section, we shall give the proof of the logarithmic decay result. To this end, we only need to prove Theorem 2.2.

Proof of Theorem 2.2 We divide the proof into two steps.

Step 1. Fix $F=\left(f^{0}, f^{1}, g^{0}, g^{1}\right) \in \mathcal{H}$ and $U_{0}=\left(y^{0}, y^{1}, z^{0}, z^{1}\right) \in D(\mathcal{A})$. Then

$$
(i \beta-\mathcal{A}) U_{0}=F
$$

is equivalent to

$$
\begin{cases}i \beta y^{0}-y^{1}=f^{0} & \text { in } \Omega, \\ \Delta^{2} y^{0}-\beta^{2} y^{0}+i \beta d y^{0}+c(x) z^{0}=f^{1}+(i \beta+d) f^{0} & \text { in } \Omega, \\ i \beta z^{0}-z^{1}=g^{0} & \text { in } \Omega, \\ \Delta^{2} z^{0}-\beta^{2} z^{0}+c(x) y^{0}=g^{1}+i \beta g^{0} & \text { in } \Omega, \\ y^{0}=\Delta y^{0}=z^{0}=\Delta z^{0}=0 & \text { on } \Gamma .\end{cases}
$$

Put

$$
p=e^{\beta s} y^{0}, \quad q=e^{\beta s} z^{0}, \quad s \in \mathbb{R} .
$$

Then $(p, q)$ solves the following equation:

$$
\begin{cases}\Delta^{2} p-p_{s s}+i d p_{s}+c(x) q=\left[f^{1}+(i \beta+d) f^{0}\right] e^{\beta s} & \text { in } \mathbb{R} \times \Omega, \\ \Delta^{2} q-q_{s s}+c(x) p=\left(g^{1}+i \beta g^{0}\right) e^{\beta s} & \text { in } \mathbb{R} \times \Omega, \\ p=\Delta p=q=\Delta q=0 & \text { on } \mathbb{R} \times \Gamma .\end{cases}
$$

Set $w=p_{s}+\Delta p$ and $z=q_{s}+\Delta q$. Clearly, $(p, w, q, z)$ solves the following equation:

$$
\begin{cases}\Delta p+p_{s}=w & \text { in } \mathbb{R} \times \Omega, \\ \Delta w-w_{s}+i d p_{s}+c(x) q=\left[f^{1}+(i \beta+d) f^{0}\right] e^{\beta s} & \text { in } \mathbb{R} \times \Omega, \\ \Delta q+q_{s}=z & \text { in } \mathbb{R} \times \Omega, \\ \Delta z-z_{s}+c(x) p=\left(g^{1}+i \beta g^{0}\right) e^{\beta s} & \text { in } \mathbb{R} \times \Omega, \\ p=w=q=z=0 & \text { on } \mathbb{R} \times \Gamma .\end{cases}
$$

Step 2. By (4.3), we have the following estimate:

$$
\left\{\begin{array}{l}
\left\|y^{0}\right\|_{H^{2}(\Omega) \cap H_{0}^{1}(\Omega)}+\left\|z^{0}\right\|_{H^{2}(\Omega) \cap H_{0}^{1}(\Omega)} \\
\quad \leq C e^{C|\beta|}\left(\|p\|_{L^{2}(X)}+\|w\|_{L^{2}(X)}+\|q\|_{L^{2}(X)}+\|z\|_{L^{2}(X)}\right) \\
\|p\|_{L^{2}(Q)}+\|w\|_{L^{2}(Q)}+\|q\|_{L^{2}(Q)}+\|z\|_{L^{2}(Q)} \\
\quad \leq C e^{C|\beta|}\left(\left\|y^{0}\right\|_{H^{2}(\Omega) \cap H_{0}^{1}(\Omega)}+\left\|z^{0}\right\|_{H^{2}(\Omega) \cap H_{0}^{1}(\Omega)}\right) \\
\|p\|_{L^{2}(\widetilde{X})} \leq C e^{C|\beta|}\left\|y^{0}\right\|_{L^{2}(\omega)} .
\end{array}\right.
$$


Applying Theorem 3.1 to Eq. (4.5), and by (4.6), we get

$$
\begin{aligned}
& \left\|y^{0}\right\|_{H^{2}(\Omega) \cap H_{0}^{1}(\Omega)}+\left\|z^{0}\right\|_{H^{2}(\Omega) \cap H_{0}^{1}(\Omega)} \\
& \quad \leq C e^{C|\beta|}\left(\left\|f^{0}\right\|_{L^{2}(\Omega)}+\left\|f^{1}\right\|_{L^{2}(\Omega)}+\left\|g^{0}\right\|_{L^{2}(\Omega)}+\left\|g^{1}\right\|_{L^{2}(\Omega)}+\left\|d y^{0}\right\|_{L^{2}(\Omega)}\right) .
\end{aligned}
$$

Multiplying the second equation of (4.2) by $\overline{y^{0}}$ and integrating it on $\Omega$, we have

$$
\int_{\Omega}\left(\left|\Delta y^{0}\right|^{2}-\beta^{2}\left|y^{0}\right|^{2}+i \beta d\left|y^{0}\right|^{2}+c z^{0} \overline{y^{0}}\right) d x=\int_{\Omega}\left[f^{1}+(i \beta+d) f^{0}\right] \overline{y^{0}} d x .
$$

Multiplying the fourth equation of (4.2) by $\overline{z^{0}}$ and integrating it on $\Omega$, we find that

$$
\int_{\Omega}\left(\left|\Delta z^{0}\right|^{2}-\beta^{2}\left|z^{0}\right|^{2}+c y^{0} \overline{z^{0}}\right) d x=\int_{\Omega}\left(g^{1}+i \beta g^{0}\right) \overline{z^{0}} d x .
$$

Taking the imaginary part in both sides of (4.8) and (4.9), we have

$$
\begin{aligned}
& |\beta| \int_{\Omega} d\left|y^{0}\right|^{2} d x \\
& \leq \quad C(1+|\beta|)\left(\left\|f^{0}\right\|_{L^{2}(\Omega)}+\left\|f^{1}\right\|_{L^{2}(\Omega)}+\left\|g^{0}\right\|_{L^{2}(\Omega)}+\left\|g^{1}\right\|_{L^{2}(\Omega)}\right) \\
& \quad \times\left(\left\|y^{0}\right\|_{L^{2}(\Omega)}+\left\|z^{0}\right\|_{L^{2}(\Omega)}\right) .
\end{aligned}
$$

As $0 \in \rho(\mathcal{A})$ (see the proof of Proposition 2.1), one can find a positive number $\delta>0$ such that $(-\delta, \delta) \subset \rho(\mathcal{A})$. For $|\beta|<\delta$, Theorem 2.2 holds trivially. For $|\beta| \geq \delta$, combining (4.7) and (4.10), we derive that

$$
\begin{aligned}
& \left\|y^{0}\right\|_{H^{2}(\Omega) \cap H_{0}^{1}(\Omega)}+\left\|z^{0}\right\|_{H^{2}(\Omega) \cap H_{0}^{1}(\Omega)} \\
& \quad \leq C e^{C|\beta|}\left(\left\|f^{0}\right\|_{L^{2}(\Omega)}+\left\|f^{1}\right\|_{L^{2}(\Omega)}+\left\|g^{0}\right\|_{L^{2}(\Omega)}+\left\|g^{1}\right\|_{L^{2}(\Omega)}\right) .
\end{aligned}
$$

Recalling that $y^{1}=i \beta y^{0}-f^{0}, z^{1}=i \beta z^{0}-g^{0}$, it follows

$$
\begin{aligned}
& \left\|y^{1}\right\|_{L^{2}(\Omega)}+\left\|z^{1}\right\|_{L^{2}(\Omega)} \\
& \quad \leq\left\|f^{0}\right\|_{L^{2}(\Omega)}+|\beta|\left\|y^{0}\right\|_{L^{2}(\Omega)}+\left\|g^{0}\right\|_{L^{2}(\Omega)}+|\beta|\left\|z^{0}\right\|_{L^{2}(\Omega)} \\
& \quad \leq C e^{C|\beta|}\left(\left\|f^{0}\right\|_{L^{2}(\Omega)}+\left\|f^{1}\right\|_{L^{2}(\Omega)}+\left\|g^{0}\right\|_{L^{2}(\Omega)}+\left\|g^{1}\right\|_{L^{2}(\Omega)}\right) .
\end{aligned}
$$

By (4.11)-(4.12), we know that there exists $C>0$ such that

$$
\left\|U_{0}\right\|_{\mathcal{H}} \leq C e^{C|\beta|}\left\|(i \beta-\mathcal{A}) U_{0}\right\|_{\mathcal{H}} .
$$

Since $\mathcal{A}$ has compact resolvents, $i \beta \in \rho(\mathcal{A})$ as long as $i \beta-\mathcal{A}$ is injective (see [20, Theorem 6.29]). Therefore, by (4.13) we have $i \mathbb{R} \subset \rho(\mathcal{A})$ and

$$
\left\|(i \beta-\mathcal{A})^{-1}\right\|_{\mathcal{L}(\mathcal{H})} \leq C e^{C|\beta|}
$$

This completes the proof of Theorem 2.2. 
Acknowledgements

The author would like to thank Professor Xiaoyu Fu for her kind guidance.

\section{Funding}

This work is partially supported by the NSF of China under grant 11971333, 11931011, the NSFC-CNRS Joint Research Project under grant 11711530142, and the PCSIRT under grant IRT 16R53.

\section{Availability of data and materials}

Not applicable.

\section{Competing interests}

The authors declare that they have no competing interests.

\section{Authors' contributions}

The author read and approved the final manuscript.

\section{Publisher's Note}

Springer Nature remains neutral with regard to jurisdictional claims in published maps and institutional affiliations.

Received: 3 January 2020 Accepted: 1 May 2020 Published online: 24 May 2020

\section{References}

1. Akil, M., Wehbe, A.: Stabilization of multidimensional wave equation with locally boundary fractional dissipation law under geometric conditions. Math. Control Relat. Fields 9, 97-116 (2019)

2. Alabau, F.: Indirect boundary stabilization of weakly coupled hyperbolic systems. SIAM J. Control Optim. 41, 511-541 (2002)

3. Alabau, F., Cannarsa, P., Komornik, V.: Indirect internal stabilization of weakly coupled systems. J. Evol. Equ. 2, 127-150 (2002)

4. Alabau, F., Léautaud, M.: Indirect stabilization of locally coupled wave-types equations. ESAIM Control Optim. Calc. Var. 18, 548-582 (2010)

5. Ammari, K., Hassine, F., Robbiano, L.: Stabilization for vibrating plate with singular structural damping (2019) arXiv:1905.13089

6. Bardos, C., Lebeau, G., Rauch, J.: Sharp sufficient conditions for the observation, control and stabilization from the boundary. SIAM J. Control Optim. 30, 1024-1165 (1992)

7. Batty, C.J.K., Duyckaerts, T.: Non-uniform stability for bounded semi-groups on Banach spaces. J. Evol. Equ. 8, 765-780 (2008)

8. Burq, N.: Décroissance de l'énergie locale de l'équation des ondes pour le probléme extérieur et absence de résonance au voisinagage du réel. Acta Math. 180, 1-29 (1998)

9. Cornilleau, P., Robbiano, L.: Carleman estimates for the Zaremba boundary condition and stabilization of waves. Am. J. Math. 136, 393-444 (2014)

10. Dell'Oro, F., Rivera, J.E.M., Pata, V.: Stability properties of an abstract system with applications to linear thermoelastic plates. J. Evol. Equ. 13, 777-794 (2013)

11. Duyckaerts, T:: Optimal decay rates of the energy of a hyperbolic-parabolic system coupled by an interface. Asymptot. Anal. 51, 17-45 (2007)

12. Fu, X.: Sharp decay rates for the weakly coupled hyperbolic system with one internal damping. SIAM J. Control Optim 50, 1643-1660 (2012)

13. Fu, X., Lü, Q.: Stabilization of the weakly coupled wave-plate system with one internal damping (2017) arXiv:1801.00232

14. Fursikov, A.V., Imanuvilov, O.Y.: Controllability of Evolution Equations. Lecture Notes Series, vol. 34. Research Institute of Mathematics, Seoul National University, Seoul (1994)

15. Guzmán, R.B., Tucsnak, M.: Energy decay estimates for the damped plate equation with a local degenerated dissipation. Syst. Control Lett. 48, 191-197 (2003)

16. Hajej, A., Hajjej, Z., Tebou, L.: Indirect stabilization of weakly coupled Kirchhoff plate and wave equations with frictional damping. J. Math. Anal. Appl. 474, 290-308 (2019)

17. Han, Z., Liu, Z.: Regularity and stability of coupled plate equations with indirect structural or Kelvin-Voigt damping ESAIM Control Optim. Calc. Var. 25, 51 (2019)

18. Hao, J., Liu, Z., Yong, J.: Regularity analysis for an abstract system of coupled hyperbolic and parabolic equations. J. Differ. Equ. 259, 4763-4798 (2015)

19. Hassine, F.: Logarithmic stabilization of the Euler-Bernoulli transmission plate equation with locally distributed Kelvin-Voigt damping. J. Math. Anal. Appl. 455, 1765-1782 (2017)

20. Kato, T: Perturbation Theory for Linear Operators. Springer, Berlin (1995) reprint of the 1980 edition

21. Lasiecka, I.: Boundary stabilization of a 3-dimensional structural acoustic model. J. Math. Pures Appl. 78, 203-232 (1999)

22. Lebeau, G.: Equation des ondes amorties. In: Algebraic and Geometric Methods in Mathematical Physics, Kaciveli, 1993, pp. 73-109. Kluwer Academic, Dordrecht (1996)

23. Liu, Z., Rao, B.: Frequency domain approach for the polynomial stability of a system of partially damped wave equations. J. Math. Anal. Appl. 335, 860-881 (2007)

24. Mansouri, S.: Boundary stabilization of coupled plate equations. Palest. J. Math. 2, 233-242 (2013)

25. Oquendo, H.P., Suárez, F.M.S.: Exact decay rates for coupled plates with partial fractional damping. Z. Angew. Math. Phys. 70, 70-88 (2019)

26. Pazy, A.: Semigroups of Linear Operators and Applications to Partial Differential Equations. Springer, New York (1983) 
27. Rao, B.: On the sensitivity of the transmission of boundary dissipation for strongly coupled and indirectly damped systems of wave equations. Z. Angew. Math. Phys. 70, 25 (2019)

28. Russell, D.L.: A general framework for the study of indirect damping mechanisms in elastic systems. J. Math. Anal. Appl. 173, 339-358 (1993)

29. Suárez, F.M.S., Oquendo, H.P.: Optimal decay rates for partially dissipative plates with rotational inertia. Acta Appl. Math. (2019). https://doi.org/10.1007/s10440-019-00259-z

30. Tebou, L.: Energy decay estimates for some weakly coupled Euler-Bernoulli and wave equations with indirect damping mechanisms. Math. Control Relat. Fields 2, 45-60 (2012)

Submit your manuscript to a SpringerOpen ${ }^{\odot}$ journal and benefit from:

- Convenient online submission

Rigorous peer review

- Open access: articles freely available online

- High visibility within the field

- Retaining the copyright to your article

Submit your next manuscript at $\gg$ springeropen.com 